\title{
A humeral hemiarthroplasty with biologic resurfacing of the glenoid using an allo-Achilles tendon: two case reports
}

\author{
Ki-Yong Choi ${ }^{1}$, Kyu-Hak Jung ${ }^{2}$, Young-Kyu Kim ${ }^{2}$, Young-Hyun Yoon ${ }^{2}$ \\ ${ }^{1}$ Department of Orthopedic Surgery, Kangwon National University Hospital, Chuncheon, Korea \\ ${ }^{2}$ Department of Orthopedic Surgery, Gil Medical Center, Gachon University College of Medicine, Incheon, Korea
}

\begin{abstract}
A hemiarthroplasty with biologic resurfacing of the glenoid is one procedure that can be performed in young patients where total shoulder arthroplasty may be difficult. The authors introduced two cases in which this procedure was performed. This approach is one treatment option for young glenoid humeral arthritis patients that addresses some of the shortcomings of an isolated hemiarthroplasty.
\end{abstract}

Keywords: Biologic; Resurfacing; Hemiarthroplasty

For elderly patients with end-stage glenohumeral arthritis, total shoulder arthroplasty (TSA) is the gold standard treatment option. Several treatment options exist in young patients, such as arthroscopic debridement, hemiarthroplasty with or without glenoid treatment, and TSA [1]. However, there is a high probability that a TSA will require revision surgery at some point in its lifetime, especially in young and active patients. Denard et al. [2] reported the 10-year survival rate of TSA was only $62.5 \%$ in patients aged 55 years or younger at the time of surgery. Many authors have reported that patients younger than 50 years tend to have worse clinical outcomes with TSA [3]. One other treatment option is to perform an isolated humeral hemiarthroplasty. However, it was reported that the hemiarthroplasty procedure is inferior to TSA because the former produces more pain, leads to less range of motion (ROM), and generally has a poor functional outcome [4]. Accordingly, the authors considered a technique to delay the TSA and to compensate for the shortcomings of hemi- arthroplasty by performing a hemiarthroplasty with biologic resurfacing of the glenoid in two patients. One case involved severe joint destruction due to rheumatoid arthritis, and the other case presented with osteoarthritis due to infection sequelae. This report details both patients' clinical and radiologic outcomes.

\section{CASE REPORT}

This study was approved by the Institutional Review Board of Gil Medical Center, Gachon University (IRB No. GAIRB2020-405). Owing to the retrospective design, the requirement for informed consent was waived.

\section{Case 1}

A 50-year-old female patient complained of pain in the left shoulder joint and difficulty with active forward elevation (aFE) for the last 4 years that had worsened about 1 year ago. The pa-

Received: October 28, 2020 Revised: January 13, $2021 \quad$ Accepted: January 14, 2021

Correspondence to: Kyu-Hak Jung

Department of Orthopedic Surgery, Gil Medical Center, Gachon University College of Medicine 21 Namdong-daero 774beon-gil, Namdong-gu, Incheon 21565, Korea

Tel: +82-32-460-3384, Fax: +82-32-460-3383, E-mail: jjangumom52@gmail.com, ORCID: https://orcid.org/0000-0003-0211-8005

Financial support: None.

Conflict of interest: None.

Copyright@ 2021 Korean Shoulder and Elbow Society. All Rights Reserved.

This is an Open Access article distributed under the terms of the Creative Commons Attribution Non-Commercial License (http://creativecommons.org/licenses/by-nc/4.0/) which permits unrestricted non-commercial use, distribution, and reproduction in any medium, provided the original work is properly cited. 
tient had been diagnosed with rheumatoid arthritis 10 years ago and had been prescribed medications, such as methotrexate and corticosteroids. The patient did not show symptom improvement despite conservative treatments, such as steroid injections, nonsteroidal anti-inflammatory drugs, and analgesics at other clinics for more than 1 year.

Upon physical examination, the aFE of the left shoulder joint was $30^{\circ}$; active external rotation at the side (aERs) was $20^{\circ}$, and active internal rotation to the posterior (aIRp) was at buttock level. Plain radiography revealed a stage 2 level of rheumatoid arthritis of the shoulder joint and also humeral head destruction according to Levigne and Franceshi's classification (Fig. 1) [5]. Magnetic resonance imaging (MRI) showed a partial tear of grade 2 of the subscapularis and supraspinatus tendon, according to Ellman's classification and 32\% supraspinatus muscle atrophy (Fig. 2) [6]. Hemiarthroplasty with biologic resurfacing of the glenoid was performed due to the patient's young age, and the integrity of the cuff was maintained despite the partial tear. The visual analog scale (VAS) pain score had significantly improved from 10 to 1 at 6 weeks postoperatively. Six months after surgery, the aFE was $145^{\circ}$, the aERs was $60^{\circ}$, and the aIRp was at the T12 level, which was no different than the patient's right shoulder joint. The same ROM was maintained at 4 years postoperatively.
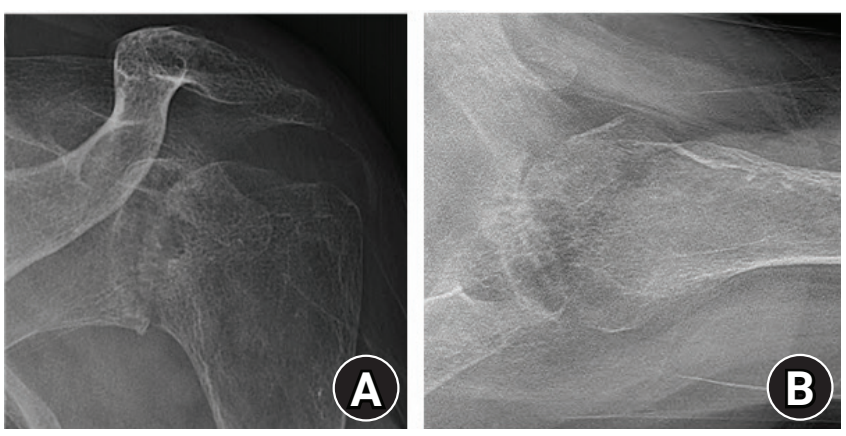

Fig. 1. The preoperative radiographs of case 1. (A) The anterior-posterior view. (B) The axial view.
At the final follow-up visit, the pain was rated as a VAS score of 0 . A joint space of $3 \mathrm{~mm}$ or greater was maintained even on the final plain radiographs, and complications such as stem loosening and infection were not observed (Fig. 3).

\section{Case 2}

A 52-year-old male patient complained of severe pain in both shoulders and visited the emergency department with a fever of $39^{\circ} \mathrm{C}$. The patient had undergone an open rotator cuff repair of the left shoulder 3 years previously and until recently had received several steroid injections at other hospitals due to multiple joint pain. ROM on both shoulders could not be confirmed due to pain, and local heat and swelling were observed. Laboratory blood testing revealed a prominent acute infection based on an elevated C-reactive protein (CRP) of $28.68 \mathrm{mg} / \mathrm{dL}$ (normal range, $0-0.5 \mathrm{mg} / \mathrm{dL}$ ) and a procalcitonin of $8.06 \mathrm{ng} / \mathrm{mL}$ (normal range, 0-0.04 ng/mL). Enhanced MRI revealed acute pyogenic arthritis in both shoulders, and arthroscopic debridement was performed on both shoulders. Afterwards, the infection status of the right shoulder improved, but the left shoulder still showed severe pain and signs of infection. Two months later, additional enhanced MRI images showed progression of osteomyelitis of the humeral head of the left shoulder (Fig. 4). Open debridement, a resection of the humeral head, and prosthesis with antibiotic-loaded acryl-
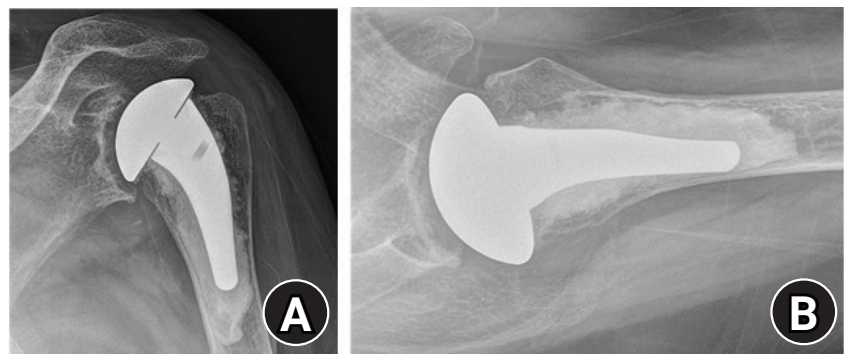

Fig. 3. The radiographs of case 1 more than 4 years postoperatively. (A) The anterior-posterior view. (B) The axial view.
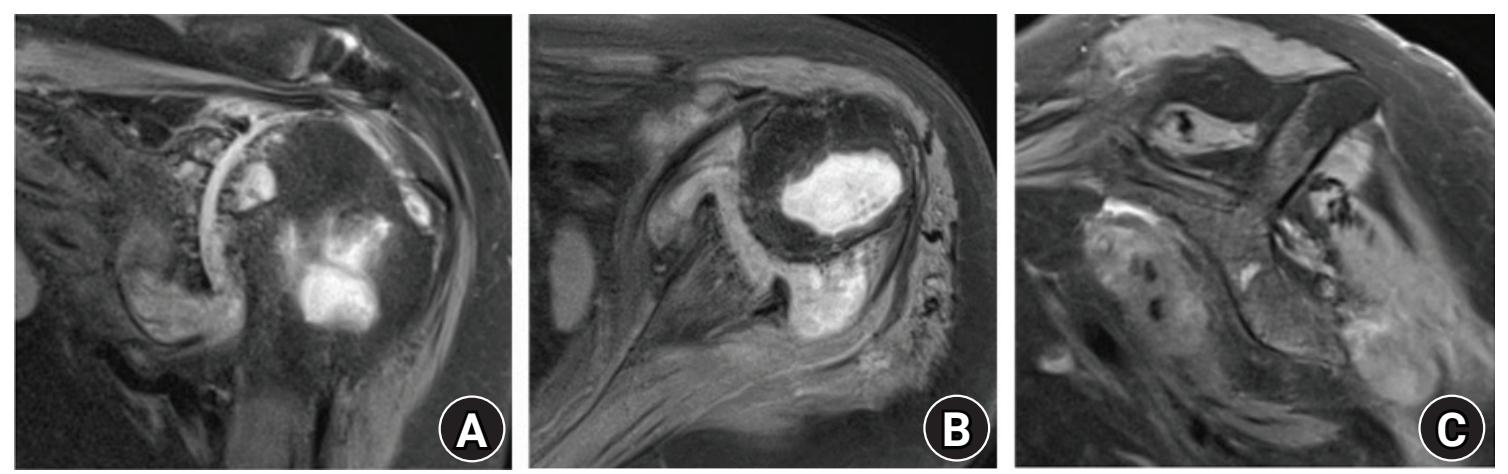

Fig. 2. Preoperative magnetic resonance images of case 1. (A) The oblique-coronal view. (B) The axial view. (C) The oblique-sagittal view. 

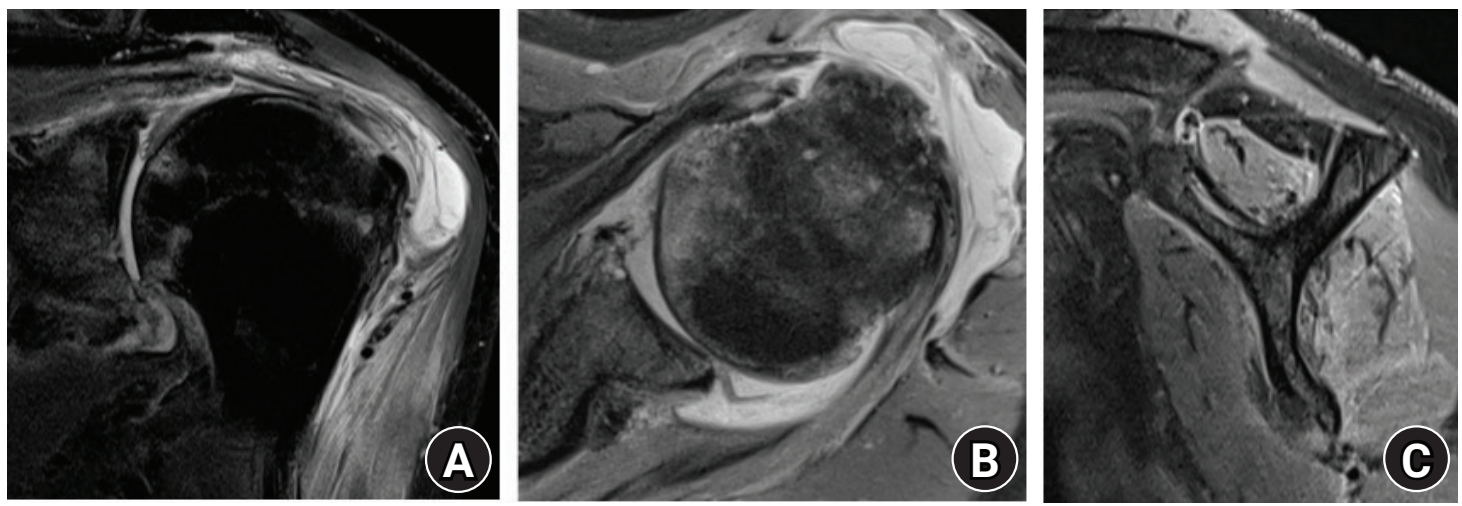

Fig. 4. Preoperative magnetic resonance images of case 2. (A) The oblique-coronal view. (B) The axial view. (C) The oblique-sagittal view.

ic cement (PROSTALAC) insertion were performed. Afterwards, the signs of infection in the left shoulder improved, but joint destruction of the left shoulder gradually progressed on plain radiography, and the patient's pain worsened. Six months after PROSTALAC insertion, hemiarthroplasty with biologic resurfacing of the glenoid was planned because the patient was highly active and young (Fig. 5). At the time of surgery, no infection ( $\leq 5$ polymorphic neutrophils per high-power field) was found on the frozen section near the glenoid and humerus, and the operation proceeded as planned. After surgery, the patient's left shoulder pain improved from a VAS of 10 preoperatively to a VAS of 3 postoperatively. Three months preoperatively, the aFE was $130^{\circ}$, the aERs was at $60^{\circ}$, and the aIRp was at the L2 level. However, the pain increased 6 months after surgery; at the final follow-up visit 2 years postoperatively, the VAS was 6 , aFE was $90^{\circ}$, aER was $40^{\circ}$, and aIRp was at the L4 level. There were no prominent infection signs, such as fever, swelling, redness, and local heat, but the CRP continued to be maintained at $1-2 \mathrm{mg} / \mathrm{dL}$ (normal range, $0-0.5 \mathrm{mg} / \mathrm{dL}$ ). In addition, stem loosening or bony erosion was not noted, and a joint space of $3 \mathrm{~mm}$ or greater was maintained (Fig. 6).

\section{Surgical Procedures and Rehabilitation}

Patients underwent the procedure in the $30^{\circ}$ beach chair position under general anesthesia. A delto-pectoral approach was used in all cases. The subscapularis was detached from the lesser tuberosity. No lesser tuberosity osteotomies were performed. The humeral head was cut with an oscillating saw along the anatomic neck. Before the operation, the diameter of the humeral head was calibrated on plain radiographs, and the size of the humeral head of the implant was confirmed using the resected head of the humerus in the operative field.

During the glenoid procedure, the soft tissue and capsule around the glenoid were carefully released to preserve the la-
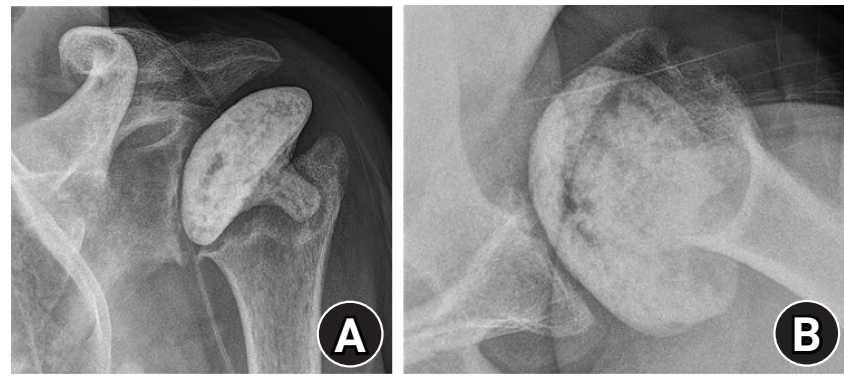

Fig. 5. The preoperative radiographs of case 2. (A) The anterior-posterior view. (B) The axial view.
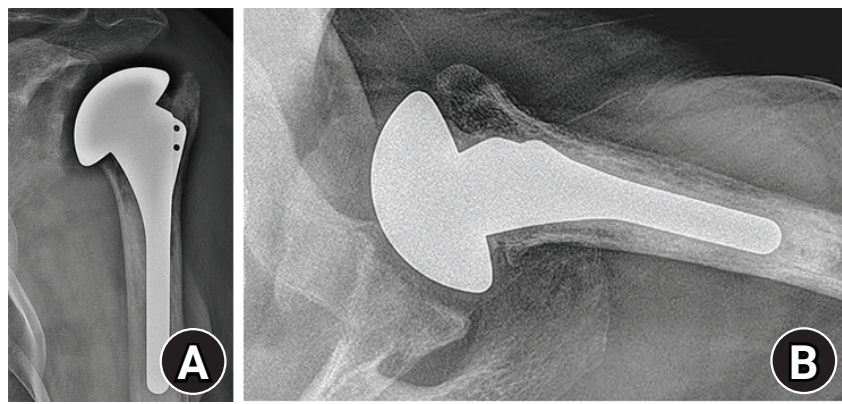

Fig. 6. The radiographs of case 22 years postoperatively. (A) The anterior-posterior view. (B) The axial view.

brum, and the glenoid was finally exposed. Curettage of the cartilage of the glenoid and multiple drilling procedures were performed. Four double-loaded absorbable suture anchors with nonabsorbable sutures were inserted into four edges of the glenoid surface (at the 12, 3, 6, and 9 oclock positions) (Fig. 7A). Next, the allo-Achilles tendon graft was prepared. The $15-\mathrm{cm} \times 3-$ $\mathrm{cm}$ raw tendon was folded and made into a final $5-\mathrm{cm} \times 3-\mathrm{cm}$ graft, and the edge of the tendon was sutured using the Krackow technique with a non-absorbable suture (Fig. 7B). The final prepared graft was approximately 5-7 mm thick. The graft was passed in a horizontal mattress fashion through all the sutures from the suture anchors (Fig. 7C). After inserting the graft into the glenoid 

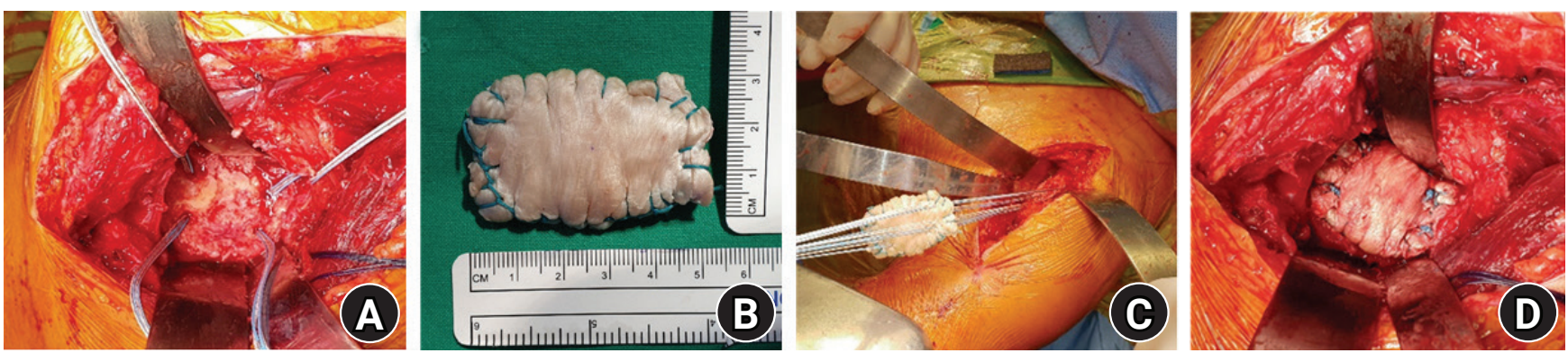

Fig. 7. (A) Preparation of the glenoid and insertion of four double-loaded absorbable anchors with nonabsorbable sutures. (B) The final appearance of the prepared allo-Achilles tendon graft. (C) The graft was passed in a horizontal mattress fashion through all the sutures. (D) The final seating of the graft in the shoulder.

while maintaining even tension, all sutures were tied. The remaining edges of the graft were sutured to the labrum using a simple suture technique with an absorbable suture (Fig. 7D).

Next, hemiarthroplasty of the humeral side was performed. The humeral canal was prepared and trialed to determine the most appropriate size and version. The best humeral component was then implanted, and the subscapularis was attached to the lesser tuberosity using a transosseous suture technique. Routine wound closure was completed, and drains were placed. After the surgery, an abductor brace was applied for 6 weeks. On the first postoperative day, passive pendulum exercises and isometric exercises were performed. Three weeks after the surgery, passive shoulder motion was started with gradual increases in the ROM. Active ROM exercises were performed from the 6th week postoperatively. Strengthening exercises were allowed to begin at 12 weeks postoperatively.

\section{DISCUSSION}

This case report described two cases of hemiarthroplasty with biologic resurfacing using an Achilles tendon allograft. Glenohumeral arthritis in young and active patients remains a challenge. For end-stage glenohumeral arthritis, the best option for surgical treatment is TSA. However, this procedure may be contraindicated in young and active patients due to concerns about the high risk of revision surgery due to glenoid component loosening and polyethylene wear or dissociation. Traditionally, a hemiarthroplasty alone has been the most common surgical procedure performed in this young and active patient group. However, longterm follow-up results have shown progressive glenoid erosion, and arthritis may also occur [3].

A hemiarthroplasty with biologic resurfacing was developed due to the risk of progressive arthritis or erosion at the glenoid. Glenoid biological resurfacing with autogenous grafts (including the anterior shoulder capsule, autogenous fascia lata, and also al- lografts including the Achilles tendon [7], and the lateral meniscus [8]) have been used as an interpositional material on the glenoid. Burkhead and Hutton [9] reported good results in a group of 14 young and active patients with end-stage glenohumeral arthritis following anterior capsule or fascia lata autograft placement to cover the glenoid. Krishnan et al. [7] carried out biological resurfacing with hemiarthroplasty. In terms of pain relief and functional recovery, they confirmed the durability of this procedure and obtained satisfactory results in $91 \%$ cases (31/34) during 2-15 years of follow-up. Their study recommended an Achilles tendon allograft as the preferred resurfacing material. Nicholson et al. [8] used a lateral meniscal allograft for a hemiarthroplasty in 30 shoulders and followed their patients for one to 4 years. Pain, ROM, and American Shoulder and Elbow Surgeons scores all improved. However, there was a $17 \%$ complication rate. Some authors feel that this lateral meniscus approach is more suitable for load sharing and load bearing. Strauss et al. [10] reported a high rate of clinical failure using a lateral meniscus allograft or human acellular dermal tissue matrix for biologic glenoid resurfacing. This surgical technique has been used in several ways and with a variety of graft materials. However, the best biological resurfacing tissues and healing potentials have yet to be demonstrated. Some investigators have reported favorable long-term results, although others have found this procedure unreliable.

In the present study, we used an Achilles tendon allograft for biologic resurfacing and achieved satisfactory results in a rheumatoid arthritis patient but an unsatisfactory outcome in the case of infection sequela. The satisfactory outcomes are thought to be due to restoring a concentric glenohumeral articulation while preserving the glenoid bone stock. The latter result was likely caused by adhesion of infected tissues and any remaining uncured chronic, low-grade infection. Nevertheless, the advantage of biological resurfacing with hemiarthroplasty is to provide an instant smooth surface and pain relief in young patients without 
the complications of TSA, such as glenoid bone loss and implant loosening. Thus, conversion to a TSA procedure may be relatively uncomplicated.

Biological resurfacing is a good treatment option for end-stage glenohumeral arthritis patients who wish to delay TSA and return to active sports or manual labor. More prospective and longer-term studies are necessary to establish a clear criterion for using hemiarthroplasty with biologic resurfacing for young patients with glenohumeral arthritis. However, in limited cases, this procedure may be one of the best treatment options.

\section{ORCID}

$\begin{array}{ll}\text { Ki-Yong Choi } & \text { https://orcid.org/0000-0001-6091-1891 } \\ \text { Kyu-Hak Jung } & \text { https://orcid.org/0000-0003-0211-8005 } \\ \text { Young-Kyu Kim } & \text { https://orcid.org/0000-0001-5672-505X } \\ \text { Young-Hyun Yoon } & \text { https://orcid.org/0000-0002-3977-9037 }\end{array}$

\section{REFERENCES}

1. Saltzman BM, Leroux TS, Verma NN, Romeo AA. Glenohumeral osteoarthritis in the young patient. J Am Acad Orthop Surg 2018;26:e361-70.

2. Denard PJ, Raiss P, Sowa B, Walch G. Mid- to long-term follow-up of total shoulder arthroplasty using a keeled glenoid in young adults with primary glenohumeral arthritis. J Shoulder
Elbow Surg 2013;22:894-900.

3. Sperling JW, Cofield RH, Rowland CM. Neer hemiarthroplasty and Neer total shoulder arthroplasty in patients fifty years old or less: long-term results. J Bone Joint Surg Am 1998;80:464-73.

4. Gartsman GM, Roddey TS, Hammerman SM. Shoulder arthroplasty with or without resurfacing of the glenoid in patients who have osteoarthritis. J Bone Joint Surg Am 2000;82:26-34.

5. Lévigne C, Franceschi JP. Rheumatoid arthritis of the shoulder: radiological presentation and results of arthroplasty. Berlin: Springer; 1999. p. 221-30.

6. Ellman H. Rotator cuff disorders. Philadelphia, PA: Lea \& Febiger; 1993. p. 98-119.

7. Krishnan SG, Nowinski RJ, Harrison D, Burkhead WZ. Humeral hemiarthroplasty with biologic resurfacing of the glenoid for glenohumeral arthritis: two to fifteen-year outcomes. J Bone Joint Surg Am 2007;89:727-34.

8. Nicholson GP, Goldstein JL, Romeo AA, et al. Lateral meniscus allograft biologic glenoid arthroplasty in total shoulder arthroplasty for young shoulders with degenerative joint disease. J Shoulder Elbow Surg 2007;16(5 Suppl):S261-6.

9. Burkhead WZ Jr, Hutton KS. Biologic resurfacing of the glenoid with hemiarthroplasty of the shoulder. J Shoulder Elbow Surg 1995;4:263-70.

10. Strauss EJ, Verma NN, Salata MJ, et al. The high failure rate of biologic resurfacing of the glenoid in young patients with glenohumeral arthritis. J Shoulder Elbow Surg 2014;23:409-19. 